\title{
TROMBOFLEBITE SÉPTICA DA VEIA PORTA SECUNDÁRIA À APENDICITE
}

\section{Septic thrombophlebitis of the portal vein as a complication of appendicitis}

\author{
Olival Cirilo Lucena da FONSECA-NETO, Luiz Paulo Figueiredo VIEIRA, Antônio Lopes de MIRANDA
}

\begin{abstract}
Fonseca-Neto OCL, Vieira LPF, Miranda AL. Tromboflebite séptica da veia porta secundária à apendicite. ABCD Arq Bras Cir Dig 2007; 20(2):137-8.
RESUMO - Introdução - A tromboflebite séptica da veia porta ou pileflebite é evento raro e associado com alta mortalidade e seu diagnóstico requer a demonstração de trombo portal. Relato do caso - Mulher de 19 anos com história de dor abdominal há 40 dias com piora há 10 foi admitida no hospital. Referia dor de forte intensidade em hipocôndrio direito, vômitos, febre, e calafrios. No exame físico de entrada tinha sinais e sintomas de sepse abdominal. Submetida à laparotomia exploradora foi encontrado abscesso hepático e apêndice ileiocecal rôto e bloqueado. Realizada apendicectomia e drenagem de abscesso, que evoluiu com necessidade de re-laparotomia no $6^{\circ}$ dia e drenagem de novos abscessos hepáticos. Após longa internação, recebeu alta e no seguimento de 6 meses estava bem. Conclusão - A pileflebite é complicação rara, porém grave, devendo ser rapidamente diagnosticada e o seu manejo multidisciplinar instituído prontamente.

DESCRITORES - Tromboflebite. Apendicite. Abscesso hepático.
\end{abstract}

\section{INTRODUÇÃO}

A tromboflebite séptica da veia porta ou pileflebite consiste na trombose infectada e supurativa da veia porta que pode ser complicação de uma sepse abdominal de qualquer etiologia na região de drenagem da veia porta. É rara e está associada com alta mortalidade. O diagnóstico de pileflebite requer a demonstração de trombo portal em paciente febril e com bacteremia ${ }^{1}$. Como é condição incomum e os sintomas são inespecíficos, o diagnóstico tardio é freqüente ${ }^{4}$. Os autores apresentam um caso de tromboflebite séptica de veia porta com abscesso hepático causado por apendicite aguda com estágio avançado, evoluindo com choque séptico e com evolução favorável.

\section{RELATO DO CASO}

Paciente do sexo feminino, 19 anos, natural de Pernambuco, foi admitida na emergência do Hospital da Restauração com história de dor abdominal há 40 dias com piora progressiva. Referia dor de forte intensidade em hipocôndrio direito, vômitos, febre e calafrios. Ainda mencionava disúria e hematúria.

Apresentava passado cirúrgico de cesariana (há 1 ano). No exame físico de entrada tinha sinais e sintomas de sepse abdominal $(\mathrm{FC}=140 \mathrm{bpm}, \mathrm{FR}=48 \mathrm{ipm}$, peritonismo

Trabalho realizado no Serviço de Cirurgia Geral e do Trauma do Hospital da Restauração - SUS - Recife,PE, Brasil.

Endereço para correspondência: Olival Cirilo Lucena Fonseca Neto, e-mail: olivalneto@globo.com à palpação do abdome). Submetida à laparotomia exploradora foi achado abscesso hepático em segmento I e apêndice rôto e bloqueado. Foi realizada apendicectomia e drenagem de abscesso. Choque séptico refratário instalouse a partir do $1^{\circ}$ dia de pós-operatório até o $6^{\circ}$. Manejo hemometabólico foi instituído (noradrenalina, dobutamina, ventilação mecânica com $\mathrm{FiO}_{2}>60 \%$, sedação). Relaparotomia no $6^{\circ}$ dia e drenagem de novos abscessos hepáticos (segmento II e III), foram necessárias no mesmo internamento. Suporte nutricional enteral foi realizado desde o $2^{\circ}$ dia de pós-operatório. Antimicrobianos e antifúngico foram administrados baseado em culturas (Pseudomonas aeroginosa e leveduras). Ultra-som-Doppler realizado em leito flagrou transformação cavernomatosa com circulação colateral pós-trombótica. Após 62 dias de internação hospitalar, recebeu alta. Após 6 meses de acompanhamento ambulatorial a paciente ganhou $10 \mathrm{~kg}$ e apresentou estudos ecográfico e tomográfico com ausência de abscessos hepáticos, transformação cavernomatosa da veia porta completa e fluxo hepatopetal presente. A anticoagulação com Warfarin foi iniciada no $20^{\circ}$ dia de pós-operatório e permaneceu até o $6^{\circ}$ mês com controle laboratorial.

\section{DISCUSSÃO}

$\mathrm{Na}$ era pré-antibiótico a pileflebite ${ }^{1}$ era universalmente fatal. Hoje, a evolução dessa entidade, melhorou devido às modernas modalidades diagnósticas e terapêuticas. A apendicite, no passado, era a principal causa de pileflebite. Hoje, a diverticulite é o principal foco séptico abdominal 


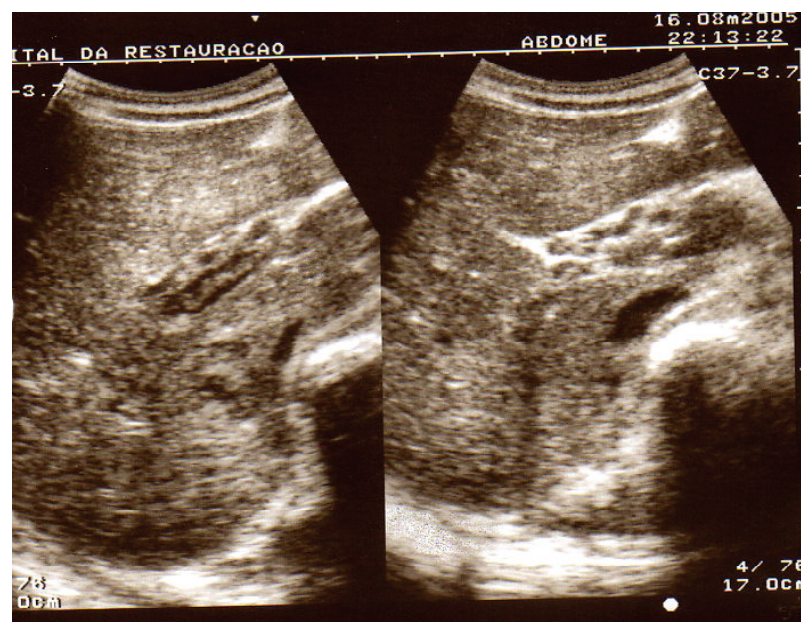

FIGURA 1 - Estudo ecográfico mostrando trombose da veia porta e abscesso hepático

que origina a tromboflebite séptica da veia porta. Doença inflamatória intestinal, perfuração intestinal, pancreatite, infecções pélvicas também podem originar o quadro séptico trombótico portal.

Pode acometer indivíduos em qualquer faixa etária (6-75 anos $)^{4}$. A apresentação clínica é inespecífica e o seu diagnóstico frequentemente tardio. Febre, dor abdominal, icterícia, náuseas e vômitos são os sintomas mais encontrados. Os exames sanguíneos poderão demonstrar infecção e disfunção hepática ${ }^{3}$. As culturas (sangue, urina) usualmente demonstram microorganismos gram-negativos (Escherichia coli, B. fragilis, Proteus mirabilis, Klebsiella pneumoniae, entre outras enterobactérias). A utilização de exames de imagem são necessários para realizar o diagnóstico ${ }^{3,4}$. O ultra-som, a tomografia e o ultra-som-Doppler são os mais usados. Os dois primeiros demonstram o foco séptico (abscesso hepático, apendicite, diverticulite,

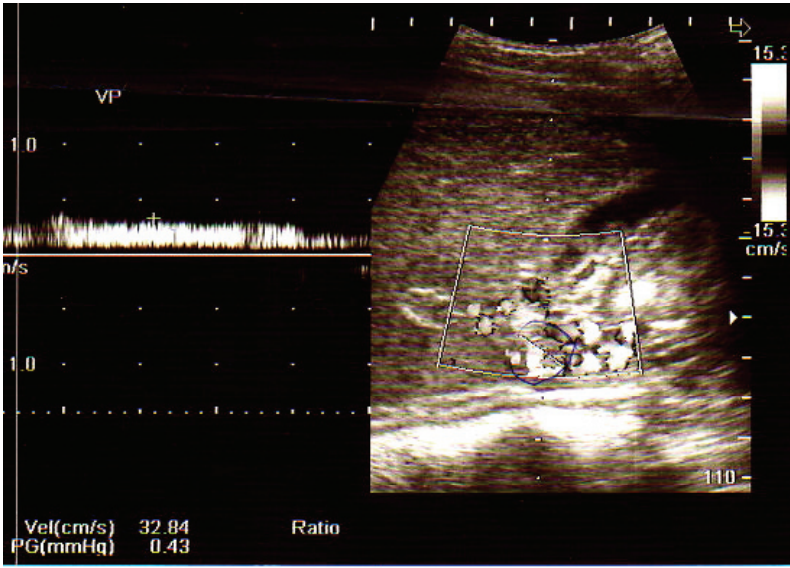

FIGURA 2 - USG-Doppler mostrando transformação cavernomatosa e circulação colateral

abscesso cavitário) e a presença do trombo. Ao Doppler o fluxo portal é demonstrado e a presença de colaterais hepatopetais e transformação cavernomatosa é procurada $a^{2,3,4}$. Uma vez estabelecido o diagnóstico, a causa deve ser tratada e o início dos antimicrobianos não é retardado. A terapia com anticoagulante ainda é controversa. No entanto, é recomendada quando existe ambiente de hipercoagulação ou quando a trombose é extensa ${ }^{3,4,5}$.

A mortalidade que era de $100 \%$ até 1946 , hoje varia de $11 \%$ a $32 \%{ }^{2}$.

\section{CONCLUSÃO}

A pileflebite é complicação rara, porém grave, devendo ser rapidamente diagnosticada e o seu manejo multidisciplinar instituído prontamente.

Fonseca-Neto OCL, Vieira LPF, Mirando AL. Septic thrombophlebitis of the portal vein as a complication of appendicitis. ABCD Arq Bras Cir Dig 2007; 20(2):137-8.

ABSTRACT - Background - Portal vein septic thrombophlebitis or pylephlebitis is a rare event associated with high mortality rates and its' diagnosis requires portal thrombosis demonstration. Case report - Nineteen year-old female was admitted to the hospital with a history of abdominal pain for the last 40 days, worsening for the last 10. The patient complained of intense and strong pain in the right hypochondrium, vomit, fever and cold chills. During physical examination the patient showed signs and symptoms of abdominal sepsis. She was then submitted to exploratory laparotomy, during which a hepatic abscess and blocked and ruptured ileocecal appendix was found. Appendicectomy and drainage of the abcess was performed, having the necessity of a second laparotomy in the sixth day and further drainage of new formatted hepatic abscesses. After a long period of admission, the patient was discharged and was well following the next six months. Conclusion - Pylephlebitis is a rare and severe complication, which has the urgency of being quickly and appropriately diagnosed as well as in a multidisciplinary manner.

HEADINGS - Thrombophlebitis. Appendicitis. Liver abscess.

\section{REFERÊNCIAS}

1. Germain MA, Soukhni N, Bouzard D. Thrombose veineuse mésentérique compliquant une appendicite aiguê. Ann Chir. 2002;127:381-4.

2. Kasper LD, Sahani D, Misdraji J. Case 25-2005: a 40-year-old man with prolonged fever and weight loss. N Engl J Med. 2005;353:713-22.

3. Mireko M, Berry PA, Brennan J, Aga R. Unrecognized pylephlebitis causing life-threatening septic shock: a case report. Word J Gastroenterol. 2005;11:6145 .

4. Nishimori H, Ezoe E, Ura H, Imaizumi H, Meguro M, Furuhata T, Katsuramaki T, Hata F, Yasoshima T, Hirata K, Asai Y. Septic thrombophlebitis of the portal and superior mesenteric veins as a complication of appendicitis: report of a case. Surg Today. 2004;34:173-6.
5. Sherigan R, Amir AK, Bobba RK, Arsura EL, Srinivas N. Abdominal pain secondary to pylephlebitis: an uncommon disease of the portal venous system, treated with local thrombolytic terapy. Dig Dis Sci. 2005;50:983-7.

Conflito de interesse: não há

Fonte financiadora: não há Recebido para publicação em: 20/02/2007 Aceito para publicação em: 06/05/2007 